\title{
Verhaltenstherapie
}

Verhaltenstherapie 1998;8:297

\section{Internationale Schizophrenie-Reintegrations-Preise vergeben}

Die Preisträger der internationalen Lilly Schizophrenia Reintegration A wards kommen in diesem Jahr aus D eutschland, Großbritannien und der Tschechischen R epublik. Insgesamt drei Projekte psychiatrischer Fachkräfte aus Klinik, Pflege und Sozialarbeit sowie ein journalistischer Beitrag wurden anläßlich der Tagung der größten psychiatrischen Berufsvereinigung, der World Psychiatric A ssociation (WPA ), in G enf gewürdigt. $M$ it der Preisverleihung ehrt das preisstiftende U nternehmen Eli Lilly gemeinsam mit der WPA herausragende therapeutische $L$ eistungen sowie beispielhafte journalistische Beiträge zur sozialen Wiedereingliederung schizophreniekranker M enschen in E uropa. Dr. G erd Schulze und seine M itarbeiter vom Verein «Psycho Soziale Integration e.V.» in Weimar erhielten den $\mathrm{H}$ auptpreis in der $\mathrm{K}$ ategorie «K linische M edizin, Pflege, Sozial- und G emeindearbeit» für inr Projekt «E ine therapeutische Woche in Tunesien». Der $\mathrm{H}$ auptpreis in der K ategorie «J ournalismus» ging an die J ournalistin I rene Stratenwerth und den Sozial psychiater D r. Thomas Bock, beide $\mathrm{H}$ amburg, für ihr K inderbuch «Die B ettelkönigin».

U m die Lilly Schizophrenia R eintegration A wards, die in diesem Jahr zum dritten $\mathrm{M}$ al ausgeschrieben wurden, hatten sich psychiatrische und soziale E inrichtungen, Therapeuten und J ournalisten aus 12 Ländern E uropas und des $N$ ahen $O$ stens beworben.

N eue Wege in der P sychiatrie: $E$ ine therapeutische Woche in Tunesien

D r. G erd Schulze, Psychologe, G ründer und Vorsitzender des Vereins «Psycho Soziale Integration e.V.» in Weimar, führte gemeinsam mit seinen Mitarbeitern ein in Deutschland einzigartiges Projekt durch: A usgehend von der Beobachtung, daß in den vergangenen Jahren bei psychisch kranken Menschen im Frühjahr und im $\mathrm{H}$ erbst psychotische $\mathrm{E}$ pisoden häufiger und stärker als zu anderen Jahreszeiten auftraten, reiste er mit acht vorwiegend schizophrenen E rkrankten im Februar 1998 für eine Woche nach Tunesien. M an wollte herausfinden, ob und wie sich der psychische Zustand bei plötzlichem Wechsel des K limas und Kulturkreises verändert. D as therapeutische Ziel, gewohnte Verhaltensweisen zu ändern und neue, positive Verhaltensreaktionen künftig im A lltag umsetzen zu können, konnte erreicht werden. In keinem Fall kam es zu psychotischen Reaktionen. Die Medikamentendosis konnte bei einigen $E$ rkrankten reduziert werden und mußte auch nach der Rückkehr nicht erhöht werden. G erd Schulze plant eine Fortführung des Projektes mit einer größeren Patientenzahl im nächsten Jahr.
Würdigung auch für deutsches $\mathrm{K}$ inderbuch

«Schizophrene M enschen können Stimmen hören, die andere nicht hören, und Bilder sehen, die andere nicht sehen. So wie fernsehen ohne Fernsehapparat oder träumen ohne Schlaf.» $M$ it diesen Worten beschreiben die $\mathrm{H}$ amburger Journalistin Irene Stratenwerth und der Sozialpsychiater Dr. Thomas Bock in ihrem Buch, das modernes Großstadtmärchen und J ugendkrimi zugleich ist, die Schizophrenie. $\mathrm{N}$ ahe an der $\mathrm{K}$ inderrealität schildern die Autoren die besondere Erlebnisweise und $G$ efährdung schizophrener $M$ enschen anhand der L ebensgeschichte einer schizophrenen Frau. Die Welt dieser Frau wird mit der kindlichen Wahrnehmungswelt in Beziehung gesetzt, ohne daß die Verfasser den pädagogischen Zeigefinger erheben.

E hrenpreise für weitere drei B ewerber aus D eutschland A lle Preisträger wurden in $\mathrm{G}$ enf für ihre Leistungen, die schizophreniekranke Menschen auf inrem Weg zur Wiedereingliederung in die Gesellschaft unterstützen, mit einem dem R eintegrationsgedanken symbolisierenden $\mathrm{K}$ ristall-O bjekt und einer $\mathrm{U}$ rkunde ausgezeichnet. $\mathrm{D}$ ie $\mathrm{G}$ ewinner aus $\mathrm{K}$ linikmedizin, Pflege und Sozialarbeit erhielten zudem einen mit 5.000 U S-D ollar dotierten G eldpreis zur U nterstützung ihrer Projektarbeit. Die unabhängige und international besetzte J ury benannte sechs weitere $\mathrm{E}$ hrenpreisträger, deren B eiträge ebenfalls eine besondere Würdigung verdienen. Die drei deutschen E hrenpreise gingen an das Z entrum für Psychiatrie Weissenau in $\mathrm{R}$ avensburg sowie an die Berliner Journalistinnen A nke Nolte (Berliner M orgenpost) und M iriam Loewy (Süddeutscher R undfunk).

Patrik Jonsson, Vertreter des Unternehmens Eli Lilly and Company, hob in Genf die großartige Leistung der Preisträger sowie ihren E insatz gegen die Diskriminierung der $\mathrm{E}$ rkrankten hervor. «Fortschritte in der B ehandlung müssen mit dem wachsenden öffentlichen und professionellen Bewußtsein und dem Verständnis für die $\mathrm{K}$ rankheit $\mathrm{H}$ and in $\mathrm{H}$ and gehen, so daß die B etroffenen die U nterstützung erhalten, die sie brauchen, um sich in die G esellschaft einzugliedern», sagte Jonsson.

Lilly - kompetenter Partner in der Therapie

Eli Lilly and Company ist ein weltweit forschendes pharmazeutisches U nternehmen mit Sitz in den USA, das durch die Lilly D eutschland $\mathrm{G} \mathrm{mbH}$ auch in Deutschland vertreten ist. Schwerpunkte des Lilly ZNS-Bereiches liegen in der Entdeckung, E rforschung und E ntwicklung hochwertiger A rzneimittel, mit denen $\mathrm{K}$ rankheiten des zentralen Nervensystems, wie zum Beispiel Schizophrenie, gelindert werden können und die Kranken helfen, ihren A lltag besser zu bewältigen. Die Lilly Schizophrenie-R eintegrations-Preise werden jährlich ausgeschrieben. 